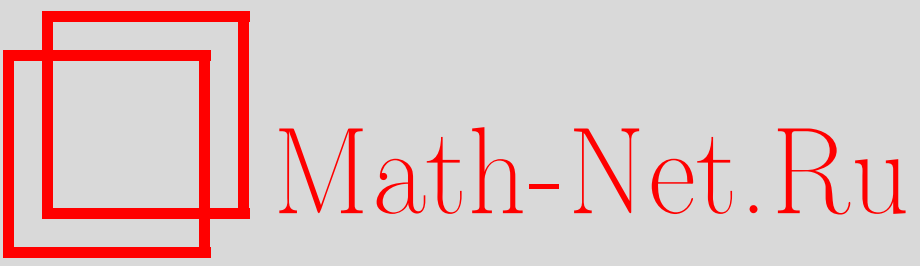

В. Ю. Королев, С. Попов, Уточнение оценок скорости сходимости в центральной предельной теореме при отсутствии моментов порядков, бо́льших второго, Теория вероятн. и ее примен., 2011, том 56, выпуск 4, 797-805

DOI: https://doi.org/10.4213/tvp4424

Использование Общероссийского математического портала Math-Net.Ru подразумевает, что вы прочитали и согласны с пользовательским соглашением

http://www.mathnet.ru/rus/agreement

Параметры загрузки:

IP: 54.196 .121 .252

26 апреля 2023 г., $15: 39: 54$ 
(C) 2011 г. КОРОЛЕВ В. Ю.

\section{УТОЧНЕНИЕ ОЦЕНОК СКОРОСТИ СХОДИМОСТИ В ЦЕНТРАЛЬНОЙ ПРЕДЕЛЬНОЙ ТЕОРЕМЕ ПРИ ОТСУТСТВИИ МОМЕНТОВ ПОРЯДКОВ, БО́ЛЬШИХ ВТОРОГО}

Уточнены верхние оценки абсолютной постоянной в оценке скорости сходимости в центральной предельной теореме в терминах урезанных моментов. Показано, что абсолютная константа в неравенстве Осипова не превосходит 2.011 .

Ключевые слова и фразы: центральная предельная теорема, оценка скорости сходимости, абсолютная константа.

1. Введение. Пусть $X_{1}, X_{2}, \ldots$ - независимые случайные величины с $\mathbf{E} X_{i}=0$ и $\mathbf{E} X_{i}^{2}<\infty$. Для $n \in \mathbf{N}$ положим $S_{n}=X_{1}+\cdots+X_{n}$. Предположим, что

$$
\mathbf{D} S_{n}=\mathbf{E} X_{1}^{2}+\cdots+\mathbf{E} X_{n}^{2}=1 .
$$

Пусть $\Phi(x)$ - стандартная нормальная функция распределения. Для $x \geqslant 0$ обозначим

$$
\begin{array}{ll}
\alpha_{n}(x)=\sum_{i=1}^{n} \mathbf{E} X_{i}^{2} \mathbf{I}\left(\left|X_{i}\right| \geqslant 1+x\right), & \alpha_{n}=\alpha_{n}(0), \\
\beta_{n}(x)=\sum_{i=1}^{n} \mathbf{E}\left|X_{i}\right|^{3} \mathbf{I}\left(\left|X_{i}\right|<1+x\right), & \beta_{n}=\beta_{n}(0) .
\end{array}
$$

В 1966 г. Л. В. Осипов [5] доказал, что существует такая конечная положительная абсолютная постоянная $C$, что

$$
\Delta_{n} \equiv \sup _{y}\left|\mathbf{P}\left(S_{n}<y\right)-\Phi(y)\right| \leqslant C\left(\alpha_{n}+\beta_{n}\right)
$$

(также см. [6, гл. V, §3, теорема 7]). В 1968 г. то же неравенство было независимо доказано В. Феллером [10], который, используя метод характеристических функций, показал, что в $(2) C \leqslant 6$.

В работах [12], [13] Л. Падитц показал, что для константы $C$ в $(2)$ справедлива оценка $C<4.77$. В 1986 г. в работе [14] он же отметил, что с учетом леммы 12.2 из монографии [1] (см. лемму 5 ниже) с помощью техники, использованной в работах [12], [13], верхнюю оценку константы $C$ можно снизить до $C<3.51$.

В 1984 г. А.Барбур и П. Холл [8] доказали неравенство (2) методом Стейна и, упоминая цитированный выше результат Феллера, констатировали, что этот метод позволил им получить лишь оценку $C \leqslant 18$ (хотя в указанной работе ими доказана

* Московский государственный университет им. М. В. Ломоносова, факультет вычислительной математики и кибернетики, Ленинские горы, 119991 Москва, Россия; Институт проблем информатики РАН, ул. Вавилова, 44, кор. 2, 119333 Москва, Россия; vkorolev@cs.msu.su

** Московский государственный университет им. М. В. Ломоносова, факультет вычислительной математики и кибернетики, Ленинские горы, 119991 Москва, Россия; popovserg@yandex.ru

1) Работа поддержана Российским фондом фундаментальных исследований (проекты 11-01-00515a и 11-07-00112a), а также Министерством образования и науки РФ в рамках ФЦП «Научные и научно-педагогические кадры инновационной России на 2009-2013 годы». 
только оценка $C \leqslant 22)$. В 2001 г. Л. Чен и К. Шао опубликовали не содержащую ссылок на упомянутые выше работы Падитца [12]-[14] работу [9], в которой с помощью метода Стейна неравенство (2) было доказано с константой $C=4.1$.

Целью данной статьи является уточнение указанных оценок константы $C$. Будет показано, что в $(2)$ в общем случае $C \leqslant 2.011$. Эта оценка также будет уточнена для некоторых частных случаев.

\section{2. Вспомогательные результаты.}

Лемма 1. Пусть $X-$ случайная величина с $\mathbf{E}|X|^{3}<\infty u \mathbf{E} X=$ а. Обозначим

$$
K=\frac{17+7 \sqrt{7}}{27}<1.3156 .
$$

Тогда

$$
\mathbf{E}|X-a|^{3} \leqslant \min \left\{K \mathbf{E}|X|^{3}, \mathbf{E}|X|^{3}+3|a| \mathbf{E} X^{2}+a^{2} \mathbf{E}|X|\right\}
$$

Д о к а з а т е л ь с т в о. С одной стороны, очевидно, что

$$
\begin{aligned}
\mathbf{E}|X-a|^{3} & =\mathbf{E}|X-a|(X-a)^{2}=\mathbf{E}|X-a|\left(X^{2}-2 a X+a^{2}\right) \\
& =\mathbf{E}|X|^{3}-2 a \mathbf{E} X|X|+a^{2} \mathbf{E}|X|+|a| \mathbf{E} X^{2}-2|a| a \mathbf{E} X+|a|^{3} \\
& =\mathbf{E}|X|^{3}+3|a| \mathbf{E} X^{2}+a^{2} \mathbf{E}|X| .
\end{aligned}
$$

С другой стороны, с использованием результата работы [11] о том, что экстремум функционала, линейного по функции распределения случайной величины $X$, при одном линейном ограничении $\mathbf{E} X=a$ моментного типа достигается на некотором двухточечном распределении (также см. [2]), в статье [4] показано, что справедливо соотношение

$$
\sup _{X: \mathbf{E}|X|^{3}<\infty} \frac{\mathbf{E}|X-a|^{3}}{\mathbf{E}|X|^{3}}=\frac{17+7 \sqrt{7}}{27}<1.3156 .
$$

Лемма доказана.

Для $x \geqslant 0, n \in \mathbf{N}$ и $i=1, \ldots, n$ обозначим

$$
Y_{i}(x)=X_{i} \mathbf{I}\left(\left|X_{i}\right|<1+x\right), \quad Y_{i}=Y_{i}(0), \quad W_{n}(x)=\sum_{i=1}^{n} Y_{i}(x), \quad W_{n}=W_{n}(0) .
$$

Так как $\mathbf{E} X_{i}=0$, то

$$
\left|\mathbf{E} X_{i} \mathbf{I}\left(\left|X_{i}\right|<1+x\right)\right|=\left|\mathbf{E} X_{i} \mathbf{I}\left(\left|X_{i}\right| \geqslant 1+x\right)\right|,
$$

а поскольку имеет место соотношение (1), то

$$
\sum_{i=1}^{n} \mathbf{E} X_{i}^{2} \mathbf{I}\left(\left|X_{i}\right|<1+x\right) \leqslant \sum_{i=1}^{n} \mathbf{E} X_{i}^{2} \leqslant 1 .
$$

Лемма 2. Пусть $K-$ число из леммь 1.

$1^{\circ}$. При любых $n \in \mathbf{N}$ u $x \geqslant 0$ для любого $p \in[1, K]$ справедливо неравенство

$$
\sum_{i=1}^{n} \mathbf{E}\left|Y_{i}(x)-\mathbf{E} Y_{i}(x)\right|^{3} \leqslant \min \left\{K \beta_{n}(x), p \beta_{n}(x)+\frac{(5-p) \alpha_{n}(x)}{1+x}\right\} .
$$

$2^{\circ}$. При любьх $n \in \mathbf{N}$ и $x \geqslant 0$ справедливы неравенства

$$
1-2 \alpha_{n}(x) \leqslant \mathbf{D} W_{n}(x) \leqslant 1 .
$$

$3^{\circ}$. Пусть $\beta_{n}=\gamma \alpha_{n}, \gamma \geqslant 0$. Тогда при любьх $n \in \mathbf{N}$ справедливо неравенство

$$
\sum_{i=1}^{n} \mathbf{E}\left|Y_{i}-\mathbf{E} Y_{i}\right|^{3} \leqslant \alpha_{n} \min \{K \gamma, \gamma+4\}
$$


Д о к а з а т е л ь с т в о. $1^{\circ}$. Согласно лемме 1 , имеем

$$
\begin{aligned}
\sum_{i=1}^{n} \mathbf{E} \mid Y_{i}(x)- & \left.\mathbf{E} Y_{i}(x)\right|^{3} \leqslant \min \left\{K \sum_{i=1}^{n} \mathbf{E}\left|X_{i}\right|^{3} \mathbf{I}\left(\left|X_{i}\right|<1+x\right), \sum_{i=1}^{n} \mathbf{E}\left|X_{i}\right|^{3} \mathbf{I}\left(\left|X_{i}\right|<1+x\right)\right. \\
& +3 \sum_{i=1}^{n}\left|\mathbf{E} X_{i} \mathbf{I}\left(\left|X_{i}\right|<1+x\right)\right| \mathbf{E} X_{i}^{2} \mathbf{I}\left(\left|X_{i}\right|<1+x\right) \\
& \left.+\sum_{i=1}^{n}\left[\mathbf{E} X_{i} \mathbf{I}\left(\left|X_{i}\right|<1+x\right)\right]^{2} \mathbf{E}\left|X_{i}\right| \mathbf{I}\left(\left|X_{i}\right|<1+x\right)\right\}
\end{aligned}
$$

С одной стороны, по неравенству Ляпунова

$$
\begin{aligned}
& \sum_{i=1}^{n}\left|\mathbf{E} X_{i} \mathbf{I}\left(\left|X_{i}\right|<1+x\right)\right| \mathbf{E} X_{i}^{2} \mathbf{I}\left(\left|X_{i}\right|<1+x\right) \\
& \quad \leqslant \sum_{i=1}^{n} \mathbf{E}\left|X_{i}\right| \mathbf{I}\left(\left|X_{i}\right|<1+x\right) \mathbf{E} X_{i}^{2} \mathbf{I}\left(\left|X_{i}\right|<1+x\right) \\
& \quad \leqslant \sum_{i=1}^{n}\left[\mathbf{E}\left|X_{i}\right|^{3} \mathbf{I}\left(\left|X_{i}\right|<1+x\right)\right]^{1 / 3}\left[\mathbf{E}\left|X_{i}\right|^{3} \mathbf{I}\left(\left|X_{i}\right|<1+x\right)\right]^{2 / 3} \\
& \quad=\sum_{i=1}^{n} \mathbf{E}\left|X_{i}\right|^{3} \mathbf{I}\left(\left|X_{i}\right|<1+x\right)=\beta_{n}(x)
\end{aligned}
$$

и

$$
\begin{aligned}
\sum_{i=1}^{n} & {\left[\mathbf{E} X_{i} \mathbf{I}\left(\left|X_{i}\right|<1+x\right)\right]^{2} \mathbf{E}\left|X_{i}\right| \mathbf{I}\left(\left|X_{i}\right|<1+x\right) } \\
& \leqslant \sum_{i=1}^{n} \mathbf{E} X_{i}^{2} \mathbf{I}\left(\left|X_{i}\right|<1+x\right) \mathbf{E}\left|X_{i}\right| \mathbf{I}\left(\left|X_{i}\right|<1+x\right) \\
& \leqslant \sum_{i=1}^{n}\left[\mathbf{E}\left|X_{i}\right|^{3} \mathbf{I}\left(\left|X_{i}\right|<1+x\right)\right]^{2 / 3}\left[\mathbf{E}\left|X_{i}\right|^{3} \mathbf{I}\left(\left|X_{i}\right|<1+x\right)\right]^{1 / 3} \\
& =\sum_{i=1}^{n} \mathbf{E}\left|X_{i}\right|^{3} \mathbf{I}\left(\left|X_{i}\right|<1+x\right)=\beta_{n}(x) .
\end{aligned}
$$

С другой стороны, в силу соотношений (3) и (4) имеем

$$
\begin{aligned}
& \sum_{i=1}^{n}\left|\mathbf{E} X_{i} \mathbf{I}\left(\left|X_{i}\right|<1+x\right)\right| \mathbf{E} X_{i}^{2} \mathbf{I}\left(\left|X_{i}\right|<1+x\right) \\
& \leqslant \sum_{i=1}^{n}\left|\mathbf{E} X_{i} \mathbf{I}\left(\left|X_{i}\right|<1+x\right)\right|=\sum_{i=1}^{n}\left|\mathbf{E} X_{i} \mathbf{I}\left(\left|X_{i}\right| \geqslant 1+x\right)\right| \\
& \quad \leqslant \sum_{i=1}^{n} \mathbf{E}\left|X_{i}\right| \mathbf{I}\left(\left|X_{i}\right| \geqslant 1+x\right)=\sum_{i=1}^{n} \mathbf{E} \frac{X_{i}^{2}}{\left|X_{i}\right|} \mathbf{I}\left(\left|X_{i}\right| \geqslant 1+x\right) \\
& \leqslant \frac{1}{1+x} \sum_{i=1}^{n} \mathbf{E} X_{i}^{2} \mathbf{I}\left(\left|X_{i}\right| \geqslant 1+x\right)=\frac{\alpha_{n}(x)}{1+x}
\end{aligned}
$$

и с учетом тех же соотношений

$$
\begin{aligned}
& \sum_{i=1}^{n}\left[\mathbf{E} X_{i} \mathbf{I}\left(\left|X_{i}\right|<1+x\right)\right]^{2} \mathbf{E}\left|X_{i}\right| \mathbf{I}\left(\left|X_{i}\right|<1+x\right) \\
& \quad=\sum_{i=1}^{n}\left|\mathbf{E} X_{i} \mathbf{I}\left(\left|X_{i}\right|<1+x\right)\right| \cdot\left|\mathbf{E} X_{i} \mathbf{I}\left(\left|X_{i}\right|<1+x\right)\right| \cdot \mathbf{E}\left|X_{i}\right| \mathbf{I}\left(\left|X_{i}\right|<1+x\right)
\end{aligned}
$$




$$
\begin{aligned}
& =\sum_{i=1}^{n}\left|\mathbf{E} X_{i} \mathbf{I}\left(\left|X_{i}\right| \geqslant 1+x\right)\right| \cdot\left|\mathbf{E} X_{i} \mathbf{I}\left(\left|X_{i}\right|<1+x\right)\right| \cdot \mathbf{E}\left|X_{i}\right| \mathbf{I}\left(\left|X_{i}\right|<1+x\right) \\
& \leqslant \sum_{i=1}^{n}\left|\mathbf{E} X_{i} \mathbf{I}\left(\left|X_{i}\right| \geqslant 1+x\right)\right| \cdot\left[\mathbf{E}\left|X_{i}\right| \mathbf{I}\left(\left|X_{i}\right|<1+x\right)\right]^{2} \\
& \leqslant \sum_{i=1}^{n}\left|\mathbf{E} X_{i} \mathbf{I}\left(\left|X_{i}\right| \geqslant 1+x\right)\right| \cdot \mathbf{E} X_{i}^{2} \mathbf{I}\left(\left|X_{i}\right|<1+x\right) \\
& \leqslant \sum_{i=1}^{n}\left|\mathbf{E} X_{i} \mathbf{I}\left(\left|X_{i}\right| \geqslant 1+x\right)\right| \leqslant \sum_{i=1}^{n} \mathbf{E}\left|X_{i}\right| \mathbf{I}\left(\left|X_{i}\right| \geqslant 1+x\right) \\
& =\sum_{i=1}^{n} \mathbf{E} \frac{X_{i}^{2}}{\left|X_{i}\right|} \mathbf{I}\left(\left|X_{i}\right| \geqslant 1+x\right) \leqslant \frac{1}{1+x} \sum_{i=1}^{n} \mathbf{E} X_{i}^{2} \mathbf{I}\left(\left|X_{i}\right| \geqslant 1+x\right)=\frac{\alpha_{n}(x)}{1+x} .
\end{aligned}
$$

Таким образом, как вторая, так и третья сумма во втором выражении под знаком минимума в (5) допускает двоякую верхнюю оценку как с помощью величины $\beta_{n}(x)$, так и с помощью величины $\alpha_{n}(x) /(1+x)$. Первое утверждение леммы доказано.

$2^{\circ}$. С учетом (1) и (3) имеем

$$
\begin{aligned}
\mathbf{D} W_{n}(x) & =\sum_{i=1}^{n} \mathbf{E} X_{i}^{2} \mathbf{I}\left(\left|X_{i}\right|<1+x\right)-\sum_{i=1}^{n}\left[\mathbf{E} X_{i} \mathbf{I}\left(\left|X_{i}\right|<1+x\right)\right]^{2} \\
& =1-\sum_{i=1}^{n} \mathbf{E} X_{i}^{2} \mathbf{I}\left(\left|X_{i}\right| \geqslant 1+x\right)-\sum_{i=1}^{n}\left[\mathbf{E} X_{i} \mathbf{I}\left(\left|X_{i}\right| \geqslant 1+x\right)\right]^{2} .
\end{aligned}
$$

Поскольку обе суммы в правой части последнего соотношения неотрицательны, отсюда вытекает, что $\mathbf{D} W_{n} \leqslant 1$. Далее, продолжая (6), замечаем, что

$$
\begin{aligned}
\mathbf{D} W_{n}(x) & =1-\alpha_{n}(x)-\sum_{i=1}^{n}\left[\mathbf{E} X_{i} \mathbf{I}\left(\left|X_{i}\right| \geqslant 1+x\right)\right]^{2} \\
& \geqslant 1-\alpha_{n}(x)-\sum_{i=1}^{n} \mathbf{E} X_{i}^{2} \mathbf{I}\left(\left|X_{i}\right| \geqslant 1+x\right)=1-2 \alpha_{n}(x) .
\end{aligned}
$$

$3^{\circ}$. Принимая во внимание утверждение $1^{\circ}$ леммы и условие $\beta_{n}=\gamma \alpha_{n}$, имеем

$$
\begin{aligned}
\sum_{i=1}^{n} \mathbf{E}\left|Y_{i}-\mathbf{E} Y_{i}\right|^{3} & \leqslant \min \left\{K \beta_{n}, p \beta_{n}+(5-p) \alpha_{n}\right\} \\
& =\alpha_{n} \min \{K \gamma, p \gamma+5-p\}=\alpha_{n} \min \{K \gamma, p(\gamma-1)+5\}
\end{aligned}
$$

Функция $f(p)=p(\gamma-1)+5$ линейна по $p$. Значит, она достигает своего минимума на одном из концов отрезка рассматриваемых значений $p$. Имеем $f(1)=\gamma+4, f(K)=$ $K \gamma+5-K>K \gamma$, откуда следует утверждение леммы.

Лемма 3. $1^{\circ}$. Пусть $q>0$. Тогда

$$
\sup _{x}|\Phi(q x)-\Phi(x)| \leqslant \frac{1}{\sqrt{2 \pi e}}\left(\max \left\{q, \frac{1}{q}\right\}-1\right) .
$$

$2^{\circ}$. Пусть $a \in \mathbf{R}$. Тогда

$$
\sup _{x}|\Phi(x+a)-\Phi(x)| \leqslant \frac{|a|}{\sqrt{2 \pi}} .
$$

Д о к а з а т е л ь с т в о. Элементарное доказательство этой леммы основано на формуле Лагранжа (также см. [6, гл. V, § 3, с. 161]). 
Лемма 4. Предположим, ито $\alpha_{n} \leqslant A$ для некоторого $A \in(0,1 / 2)$. Пусть

$$
B(A)=\frac{2}{(1+\sqrt{1-2 A}) \sqrt{1-2 A}} .
$$

Тогда

$$
1 \leqslant \frac{1}{\sqrt{\mathbf{D} W_{n}}} \leqslant 1+B(A) \alpha_{n} .
$$

Д о к а з а т е л ь с т в о. Найдем наименьшее положительное число $c$, гарантирующее справедливость неравенства

$$
\frac{1}{\sqrt{1-2 t}} \leqslant 1+c t
$$

для всех $t \in(0, A]$. Очевидно, что

$$
c=\sup _{t \in(0, A]} \frac{1}{t}\left(\frac{1}{\sqrt{1-2 t}}-1\right) .
$$

Имеем

$$
\frac{1}{t}\left(\frac{1}{\sqrt{1-2 t}}-1\right)=\frac{1-(1-2 t)}{t(1+\sqrt{1-2 t}) \sqrt{1-2 t}}=\frac{2}{(1+\sqrt{1-2 t}) \sqrt{1-2 t}} .
$$

Очевидно, что правая часть монотонно возрастает по $t$ на отрезке $[0,0.5)$ и достигает своего максимального значения на интервале $(0, A] \subset[0,0.5)$ в точке $t=A$ :

$$
c=\max _{t \in(0, A]} \frac{1}{t}\left(\frac{1}{\sqrt{1-2 t}}-1\right)=\left.\frac{2}{(1+\sqrt{1-2 t}) \sqrt{1-2 t}}\right|_{t=A}=B(A) .
$$

Теперь из утверждения $2^{\circ}$ леммы 2 с учетом условия $\alpha_{n} \leqslant A$ мы получаем

$$
\frac{1}{\sqrt{\mathbf{D} W_{n}}} \leqslant \frac{1}{\sqrt{1-2 \alpha_{n}}} \leqslant 1+B(A) \alpha_{n} .
$$

Лемма доказана.

Лемма 5. Пусть $X-$ случайная величина с $\mathbf{E} X^{2}<\infty$. Тогда

$$
\sup _{x}\left|\mathbf{P}\left(\frac{X-\mathbf{E} X}{\sqrt{\mathbf{D} X}}<x\right)-\Phi(x)\right| \leqslant 0.541 .
$$

Д о к а з а т е л ь с т в о. См., например, лемму 12.2 в книге [1].

\section{3. Основные результаты.}

Теорема 1. Пусть $n \geqslant 1$, случайнье величинь $X_{1}, \ldots, X_{n}$ независимы, $\mathbf{E} X_{i}=0$ и $\mathbf{E} X_{i}^{2}<\infty, i=1, \ldots, n$, причем выполнено условие (1). Пусть $\gamma=\beta_{n} / \alpha_{n}$. Тогда существует зависящее только от $\gamma$ положительное конечное число $C_{1}(\gamma)$ такое, что $\Delta_{n} \leqslant(1+\gamma) C_{1}(\gamma) \alpha_{n}$. При этом для $C_{1}(\gamma)$ справедливы верхние оченки, приведенные в табличе 1 .

Таблица 1. Верхние оценки числа $C_{1}(\gamma)$

\begin{tabular}{||c|c||c|c||c|c||}
\hline$\gamma$ & $C_{1}(\gamma) \leqslant$ & $\gamma$ & $C_{1}(\gamma) \leqslant$ & $\gamma$ & $C_{1}(\gamma) \leqslant$ \\
\hline$\gamma \geqslant 0$ & 2.0110 & $\gamma \geqslant 1$ & 1.5953 & $\gamma \geqslant 10$ & 0.9433 \\
$\gamma \geqslant 0.1$ & 1.9735 & $\gamma \geqslant 2$ & 1.3672 & $\gamma \geqslant 100$ & 0.6086 \\
$\gamma \geqslant 0.5$ & 1.7823 & $\gamma \geqslant 5$ & 1.0908 & $\gamma \rightarrow \infty$ & 0.5600 \\
\hline
\end{tabular}

Следствие 1. В условиях теоремь 1 неравенство (2) имеет место с $C \leqslant$ 2.0110 . 
Д о к а з а т е л ь с т в о т е о р е м ы 1. Для каждого $y \in \mathbf{R}$ событие $\left\{S_{n}<\right.$ $y\}$ влечет за собой событие

$$
\left\{W_{n}<y\right\} \cup\left\{\left|X_{1}\right| \geqslant 1\right\} \cup \cdots \cup\left\{\left|X_{n}\right| \geqslant 1\right\},
$$

тогда как событие $\left\{W_{n}<y\right\}$ влечет за собой событие

$$
\left\{S_{n}<y\right\} \cup\left\{\left|X_{1}\right| \geqslant 1\right\} \cup \cdots \cup\left\{\left|X_{n}\right| \geqslant 1\right\} .
$$

Поэтому $\sup _{y}\left|\mathbf{P}\left(S_{n}<y\right)-\mathbf{P}\left(W_{n}<y\right)\right| \leqslant \sum_{i=1}^{n} \mathbf{P}\left(\left|X_{i}\right| \geqslant 1\right)$. Следовательно, для любого $y \in \mathbf{R}$

$$
\Delta_{n} \leqslant Q_{1}+Q_{2}+Q_{3}
$$

где

$$
\begin{aligned}
& Q_{1}=\sup _{y}\left|\mathbf{P}\left(\frac{W_{n}-\mathbf{E} W_{n}}{\sqrt{\mathbf{D} W_{n}}}<\frac{y-\mathbf{E} W_{n}}{\sqrt{\mathbf{D} W_{n}}}\right)-\Phi\left(\frac{y-\mathbf{E} W_{n}}{\sqrt{\mathbf{D} W_{n}}}\right)\right|, \\
& Q_{2}=\sup _{y}\left|\Phi\left(\frac{y-\mathbf{E} W_{n}}{\sqrt{\mathbf{D} W_{n}}}\right)-\Phi(y)\right|, \\
& Q_{3}=\sum_{i=1}^{n} \mathbf{P}\left(\left|X_{i}\right| \geqslant 1\right) .
\end{aligned}
$$

Рассмотрим $Q_{1}$. В силу неравенства Берри-Эссеена с наилучшей известной на сегодняшний день оценкой абсолютной константы (см. [7]) имеем

$$
Q_{1} \leqslant \frac{0.56}{\left(\mathbf{D} W_{n}\right)^{3 / 2}} \sum_{i=1}^{n} \mathbf{E}\left|Y_{i}-\mathbf{E} Y_{i}\right|^{3} .
$$

Предположим, что $\alpha_{n} \leqslant A<1 / 2$. Тогда согласно утверждениям $2^{\circ}$ и $3^{\circ}$ леммы 2

$$
Q_{1} \leqslant \frac{0.56 \min \{K \gamma, \gamma+4\} \alpha_{n}}{(1-2 A)^{3 / 2}} .
$$

Рассмотрим $Q_{2}$. Очевидно, что

$$
Q_{2} \leqslant \sup _{y}\left|\Phi\left(\frac{y}{\sqrt{\mathbf{D} W_{n}}}\right)-\Phi(y)\right|+\sup _{y}\left|\Phi\left(y-\frac{\mathbf{E} W_{n}}{\sqrt{\mathbf{D} W_{n}}}\right)-\Phi(y)\right| \equiv Q_{21}+Q_{22} .
$$

Согласно утверждению $2^{\circ}$ леммы $2, \mathbf{D} W_{n} \leqslant 1$. Поэтому в соответствии с утверждением $1^{\circ}$ леммы 3 и леммой 4 справедливо неравенство

$$
Q_{21} \leqslant \frac{1}{\sqrt{2 \pi e}}\left(\frac{1}{\sqrt{\mathbf{D} W_{n}}}-1\right) \leqslant \frac{2 \alpha_{n}}{\sqrt{2 \pi e(1-2 A)}(1+\sqrt{1-2 A})} .
$$

Рассмотрим $Q_{22}$. В силу соотношения (2) имеем

$$
\begin{aligned}
\left|\mathbf{E} W_{n}\right| & =\left|\sum_{i=1}^{n} \mathbf{E} Y_{i}\right| \leqslant \sum_{i=1}^{n}\left|\mathbf{E} X_{i} \mathbf{I}\left(\left|X_{i}\right|<1\right)\right|=\sum_{i=1}^{n}\left|\mathbf{E} X_{i} \mathbf{I}\left(\left|X_{i}\right| \geqslant 1\right)\right| \\
& \leqslant \sum_{i=1}^{n} \mathbf{E}\left|X_{i}\right| \mathbf{I}\left(\left|X_{i}\right| \geqslant 1\right) \leqslant \sum_{i=1}^{n} \mathbf{E} X_{i}^{2} \mathbf{I}\left(\left|X_{i}\right| \geqslant 1\right)=\alpha_{n} .
\end{aligned}
$$

Поэтому в силу утверждения $2^{\circ}$ леммы 2 и утверждения $2^{\circ}$ леммы 3

$$
Q_{22} \leqslant \frac{\alpha_{n}}{\sqrt{2 \pi(1-2 A)}} .
$$

Из (9) и (10) вытекает, что

$$
Q_{2} \leqslant \frac{\alpha_{n}}{\sqrt{2 \pi(1-2 A)}}\left(1+\frac{2}{\sqrt{e}(1+\sqrt{1-2 A})}\right) .
$$


Наконец, по неравенству Маркова

$$
Q_{3}=\sum_{i=1}^{n} \mathbf{P}\left(\left|X_{i}\right| \geqslant 1\right) \leqslant \sum_{i=1}^{n} \mathbf{E} X_{i}^{2} \mathbf{I}\left(\left|X_{i}\right| \geqslant 1\right)=\alpha_{n} .
$$

Таким образом, из (7), (8), (11) и (12) мы получаем

$$
\Delta_{n} \leqslant \alpha_{n}\left[1+\frac{1}{\sqrt{2 \pi(1-2 A)}}\left(1+\frac{2}{\sqrt{e}(1+\sqrt{1-2 A})}\right)+\frac{0.56 \min \{K \gamma, \gamma+4\}}{(1-2 A)^{3 / 2}}\right] .
$$

Введем функцию

$$
H_{1}(\gamma, A)=1+\frac{1}{\sqrt{2 \pi(1-2 A)}}\left(1+\frac{2}{\sqrt{e}(1+\sqrt{1-2 A})}\right)+\frac{0.56 \min \{K \gamma, \gamma+4\}}{(1-2 A)^{3 / 2}} .
$$

Для любого $0 \leqslant A<1 / 2$ справедливо неравенство

$$
\Delta_{n} \leqslant \alpha_{n} \max \left\{H_{1}(\gamma, A), \frac{0.541}{A}\right\} .
$$

Это следует из неравенства (13) в случае $\alpha_{n} \leqslant A$ и из леммы 5 в противном случае.

Таким образом, имея в виду равенство

$$
\alpha_{n}=\frac{\alpha_{n}+\gamma \alpha_{n}}{1+\gamma}=\frac{\alpha_{n}+\beta_{n}}{1+\gamma}
$$

убеждаемся, что

$$
C_{1}(\gamma) \leqslant \min _{0 \leqslant A<1 / 2} \max \left\{\frac{H_{1}(\gamma, A)}{1+\gamma}, \frac{0.541}{A(1+\gamma)}\right\} .
$$

Вычисления по последней формуле позволяют получить значения, приведенные в таблице 1. Заметим, что первая из функций аргумента $A$ внутри минимакса является возрастающей, а вторая - убывающей. Значит, значение минимакса доставляется единственным решением уравнения

$$
\frac{H_{1}(\gamma, A)}{1+\gamma}=\frac{0.541}{A(1+\gamma)}
$$

При $\gamma>13$ (имеем $\gamma+4<K \gamma$ ) обе функции являются убывающими по $\gamma$, т.е. значение минимакса убывает. И соответствующая часть таблицы 1 получается вычислением оценки $C_{1}(\gamma)$ в одной точке. Часть таблицы 1 , соответствующая $0 \leqslant \gamma \leqslant 13$, получается численной оптимизацией на конечном отрезке. Теорема доказана.

Используя текущую наилучшую верхнюю оценку абсолютной константы в неравенстве Берри-Эссеена для сумм независимых одинаково распределенных случайных величин: $C_{0} \leqslant 0.4784$ (см. [3]), по аналогии с теоремой 1 можно доказать следующее утверждение.

Теорема 2. Пусть в дополнение $\kappa$ условиям теоремь 1 случайнье величинь одинаково распределень. Тогда существует зависящее только от $\gamma$ положительное конечное число $C_{2}(\gamma)$ такое, что $\Delta_{n} \leqslant(1+\gamma) C_{2}(\gamma) \alpha_{n}$. При этом для $C_{2}(\gamma)$ справедливы верхние оценки, приведенные в табличе 2.

Таблица 2. Верхние оценки числа $C_{2}(\gamma)$

\begin{tabular}{||c|c||c|c||c|c||}
\hline$\gamma$ & $C_{2}(\gamma) \leqslant$ & $\gamma$ & $C_{2}(\gamma) \leqslant$ & $\gamma$ & $C_{2}(\gamma) \leqslant$ \\
\hline$\gamma \geqslant 0$ & 2.0110 & $\gamma \geqslant 1$ & 1.5234 & $\gamma \geqslant 10$ & 0.8430 \\
$\gamma \geqslant 0.1$ & 1.9537 & $\gamma \geqslant 2$ & 1.2820 & $\gamma \geqslant 100$ & 0.5245 \\
$\gamma \geqslant 0.5$ & 1.7272 & $\gamma \geqslant 5$ & 0.9950 & $\gamma \rightarrow \infty$ & 0.4784 \\
\hline
\end{tabular}


Д о к а з а т е л ь с т в о. Рассуждая по аналогии с доказательством теоремы 1 , несложно убедиться, что

где

$$
C_{2}(\gamma) \leqslant \min _{0 \leqslant A<1 / 2} \max \left\{\frac{H_{2}(\gamma, A)}{1+\gamma}, \frac{0.541}{A(1+\gamma)}\right\}
$$

$$
H_{2}(\gamma, A)=1+\frac{1}{\sqrt{2 \pi(1-2 A)}}\left(1+\frac{2}{\sqrt{e}(1+\sqrt{1-2 A})}\right)+\frac{0.4784 \min \{K \gamma, \gamma+4\}}{(1-2 A)^{3 / 2}} .
$$

Вычисления по указанным формулам приводят к оценкам $C_{2}(\gamma)$, объявленным в формулировке теоремы. Теорема доказана.

Теорема 3. Пусть в дополнение $\kappa$ условиям теоремь 1 случайные величины имеют симметричные распределения. Тогда существует зависящее только от $\gamma$ положительное конечное число $C_{3}(\gamma)$ такое, что $\Delta_{n} \leqslant(1+\gamma) C_{3}(\gamma) \alpha_{n}$. При этом для $C_{3}(\gamma)$ справедливы верхние оченки, приведеннье в табличе 3.

Таблица 3. Верхние оценки числа $C_{3}(\gamma)$

\begin{tabular}{||c|c||c|c||c|c||}
\hline$\gamma$ & $C_{3}(\gamma) \leqslant$ & $\gamma$ & $C_{3}(\gamma) \leqslant$ & $\gamma$ & $C_{3}(\gamma) \leqslant$ \\
\hline$\gamma \geqslant 0$ & 1.5773 & $\gamma \geqslant 1$ & 1.3046 & $\gamma \geqslant 10$ & 0.7451 \\
$\gamma \geqslant 0.1$ & 1.5754 & $\gamma \geqslant 2$ & 1.1130 & $\gamma \geqslant 100$ & 0.5825 \\
$\gamma \geqslant 0.5$ & 1.4543 & $\gamma \geqslant 5$ & 0.8745 & $\gamma \rightarrow \infty$ & 0.5600 \\
\hline
\end{tabular}

Следствие 2. В условиях теоремь 3 неравенство (2) имеет место с $C \leqslant$ 1.5773.

Д о к а з а т е л ь с т в о т е о р е м ы 3. В рассматриваемой ситуации вместо (8) имеет место неравенство

$$
Q_{1} \leqslant \frac{0.56 \beta_{n}}{(1-2 A)^{3 / 2}}
$$

a $Q_{22}=0$, поскольку $\mathbf{E} W_{n}=0$. Поэтому справедлива оценка

$$
\Delta_{n} \leqslant \alpha_{n}\left(1+\frac{2}{\sqrt{2 \pi e(1-2 A)}(1+\sqrt{1-2 A})}\right)+\frac{0.56 \beta_{n}}{(1-2 A)^{3 / 2}} .
$$

Таким образом,

где

$$
C_{3}(\gamma) \leqslant \min _{0 \leqslant A<1 / 2} \max \left\{\frac{H_{3}(\gamma, A)}{1+\gamma}, \frac{0.541}{A(1+\gamma)}\right\}
$$

$$
H_{3}(\gamma, A)=1+\frac{2}{\sqrt{2 \pi e(1-2 A)}(1+\sqrt{1-2 A})}+\frac{0.56 \gamma}{(1-2 A)^{3 / 2}} .
$$

Вычисления по указанным формулам приводят к оценкам $C_{3}(\gamma)$, объявленным в формулировке теоремы. Теорема доказана.

Теорема 4. Пусть в дополнение $к$ условиям теоремь 3 случайные величинь одинаково распределеньл. Тогда существует зависящее только от $\gamma$ положительное конечное число $C_{4}(\gamma)$ такое, ито $\Delta_{n} \leqslant(1+\gamma) C_{4}(\gamma) \alpha_{n}$. При этом для $C_{4}(\gamma)$ справедливы верхние оценки, приведеннье в табличе 4.

Таблица 4. Верхние оценки числа $C_{4}(\gamma)$

\begin{tabular}{||c|c||c|c||c|c||}
\hline$\gamma$ & $C_{4}(\gamma) \leqslant$ & $\gamma$ & $C_{4}(\gamma) \leqslant$ & $\gamma$ & $C_{4}(\gamma) \leqslant$ \\
\hline$\gamma \geqslant 0$ & 1.5655 & $\gamma \geqslant 1$ & 1.2459 & $\gamma \geqslant 10$ & 0.6681 \\
$\gamma \geqslant 0.1$ & 1.5558 & $\gamma \geqslant 2$ & 1.0453 & $\gamma \geqslant 100$ & 0.5017 \\
$\gamma \geqslant 0.5$ & 1.4075 & $\gamma \geqslant 5$ & 0.8003 & $\gamma \rightarrow \infty$ & 0.4784 \\
\hline
\end{tabular}


Следствие 3. В условиях теоремь 4 неравенство (2) имеет место с

$$
C \leqslant 1.5655 \text {. }
$$

Д оказ а т ель с т в о т е о р е м ы 4. В данном случае

$$
C_{4}(\gamma) \leqslant \min _{0 \leqslant A<1 / 2} \max \left\{\frac{H_{4}(\gamma, A)}{1+\gamma}, \frac{0.541}{A(1+\gamma)}\right\},
$$

где

$$
H_{4}(\gamma, A)=1+\frac{2}{\sqrt{2 \pi e(1-2 A)}(1+\sqrt{1-2 A})}+\frac{0.4784 \gamma}{(1-2 A)^{3 / 2}} .
$$

Вычисления по указанным формулам приводят к оценкам $C_{4}(\gamma)$, объявленным в формулировке теоремы. Теорема доказана.

Авторы выражают признательность Л. Падитцу за неоценимую библиографическую поддержку, а также Ю. С. Нефедовой и И. Г. Шевцовой за возможность ознакомиться с работой [4].

\section{СПИСОК ЛИТЕРАТУРЫ}

1. Бхаттачария P.Н., Ранга Рао Р. Аппроксимация нормальным распределением и асимптотические разложения. М.: Наука, 1982, 286 с.

2. Золотарев B. M. Современная теория суммирования независимых случайных величин. М.: Наука, 1986, 415 с.

3. Королев В.Ю., Шевцова И. Г. Уточнение неравенства Берри-Эссеена с приложениями к пуассоновским и смешанным пуассоновским случайным суммам. Обозрение прикл. и промышл. матем., 2010, т. 17, № 1, с. 25-56.

4. Нефедова Ю.С., Шевцова И. Г. О неравномерных оценках скорости сходимости в центральной предельной теореме. - Теория вероятн. и ее примен. (в печати).

5. Осипов Л. В. Уточнение теоремы Линдеберга. - Теория вероятн. и ее примен., 1966, т. 11 , в. 2 , с. $339-342$.

6. Петров В.В. Предельные теоремы для сумм независимых случайных величин. М.: Наука, 1987, 317 с.

7. Шевцова И.Г. Уточнение оценок скорости сходимости в теореме Ляпунова. Докл. РАН, 2010, т. 435, № 1, с. 26-28.

8. Barbour A.D., Hall P. Stein's method and the Berry-Esseen theorem. - Austral. J. Statist., 1984, v. 26, p. 8-15.

9. Chen L. H. Y., Shao Q. M. A non-uniform Berry-Esseen bound via Stein's method. Probab. Theory Related Fields, 2001, v. 120, № 2, p. 236-254.

10. Feller W. On the Berry-Esseen theorem. - Z. Wahrscheinlichkeitstheor. verw. Geb., 1968 , v. 10 , p. 261-268.

11. Hoeffding $W$. The extrema of the expected value of a function of independent random variables. - Ann. Math. Statist., 1955, v. 26, № 2, p. 268-275.

12. Paditz L. Bemerkungen zu einer Fehlerabschätzung im zentralen Grenzwertsatz. Wiss. Z. Hochsch. Verkehrswesen «Friedrich List» Dresden, 1980, v. 27, № 4, p. 829837.

13. Paditz L. On error-estimates in the central limit theorem for generalized linear discounting. - Math. Operationsforsch. Statist., Ser. Statist., 1984, v. 15, № 4, p. 601610 .

14. Paditz L. Über eine globale Fehlerabschätzung im zentralen Grenzwertsatz. - Wiss. Z. Hochsch. Verkehrswesen «Friedrich List» Dresden, 1986, v. 33, № 2, p. 399-404.

Поступила в редакцию 